\title{
UN ESTILO Y SU ÉPOCA. EL CASO DE LA CERÁMICA FAMABALASTO NEGRO GRABADO DEL NOROESTE ARGENTINO
}

PALAMARCZUK, VALERIA ${ }^{\mathrm{I}}$

Fecha de Defensa: 27 de mayo de 2009 - Directora: Dra. Myriam Tarragó

Jurados: Dras. Beatriz Cremonte, laura Quiroga y Verónica Williams

En este trabajo se resumen los resultados de cinco años de investigación en el campo de los estudios sobre la producción alfarera de las sociedades de época tardía en los Valles Calchaquíes, en particular en el área de Yocavil. La tesis doctoral fue financiada mediante una beca del CONICET. Una versión de la tesis se encuentra actualmente en prensa en British Archaeological Reports, Oxford, Reino Unido.

Se presenta el estudio contextual, desde una perspectiva materialista apoyada en los conceptos guía de estilo y producción, de la alfarería Famabalasto Negro Grabado de época tardía (concepto amplio que involucra los períodos Tardío, Inca e Hispano Indígena) en los Valles Calchaquíes, en especial en el valle de Yocavil o Santa María, al sur del área. Esta interesante cerámica negra y pulida, que no es muy abundante y cuyos fragmentos pueden verse hoy en día esparcidos sobre la superficie de los viejos poblados -y aún se encuentran piezas enteras en los antiguos sepulcros acompañando a los difuntos- se destaca a simple vista por sus contrastes con otras alfarerías decoradas contemporáneas y se acerca, por la configuración de sus diseños, a la estética de ciertos bienes metálicos especiales elaborados en bronce fundido: las placas circulares y las campanas o tan-tanes santamarianas. Los pensamientos que los diferentes investigadores le habían dedicado a lo largo de décadas mostraban un abanico de posiciones diversas y las ideas vigentes indicaban una falta de consenso, en particular en lo referido a su cronología (preincaica o incaica) y raíces culturales (una cerámica local de la cultura Santamariana o un elemento foráneo introducido en la región a partir de la expansión incaica).

En la arqueología argentina contamos con una rica tradición en el estudio de los pueblos prehispánicos de los Valles Calchaquíes, gracias a ello conocemos muchos de los aspectos de la vida de las sociedades tardías. No obstante continúa siendo una necesidad el lograr mejores descripciones de los diferentes elementos de la cultura material, como un primer paso necesario hacia una interpretación del rol de los objetos -y de las representaciones en ellos plasmadas- en la vida cotidiana y en la historia de los hombres y las comunidades.

El objetivo básico del enfoque contextual propuesto es el de lograr un encuadre espacial, temporal y cultural de esta manifestación estilística, para lo cual era indispensable comenzar con una caracterización intensiva de la tecnología, las formas y los diseños que definen al estilo y la identificación de patrones o recurrencias en la distribución espacial, a escala intra-sitio, local y regional, de las variantes observadas. Luego nos propusimos avanzar en el análisis de los contextos de hallazgo para evaluar tanto las diferentes situaciones de uso, como la ubicación y extensión del mismo en el eje temporal. El proyecto de

I CONiCet • Museo Etnográfico J. B. Ambrosetti, FFyl, ubA • Moreno 350, (1091) CABA

- E-MAlL: valepala@yahoo.com.ar 
estudio regional necesitaba del examen de un gran conjunto de vasijas, de la mayor cantidad de procedencias posible. Mediante una búsqueda que intentó ser exhaustiva se registró cada pieza y fragmento ubicado, ya sea a través de un reconocimiento directo, como por fotografías e ilustraciones publicadas. Se examinaron colecciones de arqueología del noroeste argentino correspondientes a veinte museos del país y del exterior. De ese modo se pudo reunir una muestra conformada por 209 vasijas, enteras o muy completas, de estilo Famabalasto Negro Grabado. La incorporación de fragmentos a la muestra, obtenidos por distintos equipos de investigación que trabajan en la región, resultó de suma importancia, ya que hay muchas localidades arqueológicas que no están representadas en colecciones de museos, pero en las que se han hallado tiestos en excavaciones o recolecciones. En conjunto se registraron así 258 vasijas en estado fragmentario. El planteo de la problemática en el marco geográfico e histórico regional y de los lineamientos conceptuales y metodológicos, junto con un esquema de la organización de la tesis, son expuestos en el Capítulo 1.

En el Capítulo 2 se presenta una historia acerca de la conceptualización del estilo en el ámbito académico de la arqueología y se compendia la información existente sobre la distribución de hallazgos de ejemplares Famabalasto Negro Grabado en el espacio regional. Para ello se recurrió a bibliografía publicada e inédita, tesis, libretas e informes de campo y laboratorio y catálogos de colecciones.

La descripción y análisis de las características particulares del estilo comienza con el abordaje de aspectos de la tecnología alfarera, considerados en el Capítulo 3. En primer término se realizaron estudios petrográficos sobre una muestra regional compuesta por 59 fragmentos Famabalasto Negro Grabado de sitios de Yocavil, Tafí y Andalgalá. Se planteó un esquema clasificatorio de pastas cerámicas que incluye hasta el momento ocho
Estándares, que representan conjuntos de vasijas con similares características de pasta y cuatro conjuntos de mayor nivel, a los que se llamó Variedades. Éstos son agrupamientos de Estándares según alguna conducta tecnológica en particular que ellos representen, por ejemplo el trabajo con barros puros o el agregado de antiplásticos de tipo clástico. El mayor nivel inclusivo en la clasificación involucra a todas las Variedades en dos Clases, según éstas tengan o no, tiesto molido incorporado. Este criterio clasificatorio se basó en el conocimiento acerca de la importancia del tiesto molido como una tradición tecnológica de larga data que caracteriza a diversos estilos alfareros tardíos en Yocavil. Luego se avanza en un estudio preliminar mediante análisis por activación neutrónica instrumental de un conjunto de vasijas Famabalasto Negro Grabado (de Yocavil), Famabalasto Negro Sobre Rojo (de Andalgalá, valle Calchaquí y Santiago del Estero) y Belén (de Hualfín y Fiambalá). Este ensayo preliminar expone la existencia de tendencias composicionales definidas para los diferentes estilos sujetos a comparación. La última contribución del capítulo sobre tecnología alfarera se orienta al estudio de la composición del relleno blanco decorativo, que es una de las características salientes del estilo. Mediante la técnica de difracción de Rayos X se estudiaron diez muestras de rellenos, los resultados indican el uso de materiales naturales como el yeso y la calcita con un mismo fin decorativo.

La clasificación descriptiva de las formas es desarrollada en el Capítulo 4 empleando propuestas de nomenclatura sistemáticas junto con los nombres tradicionales. Se reconocieron un total de 42 variantes morfológicas, clasificadas en cuatro grupos básicos: cuencos o pucos, ollas de contorno simple, ollas con borde y ollas con cuello, siendo los cuencos la forma predominante. El análisis y clasificación de los diseños se realizó integrando métodos de observación que podrían considerarse antagónicos como el análisis jerárquico -por el cual se desglosan niveles sucesivos entre segmentación del espacio de diseño, 
motivos, elementos y rellenos- y el análisis no jerárquico, que distingue esquemas -patrones de configuración y unidades de diseño- en base a la observación de repeticiones en diferentes vasijas. Se identificaron en la muestra nueve patrones de segmentación del espacio de diseño inciso y una décima variante representada por la ausencia de diseño. En los espacios de diseño se registraron 140 modelos de guardas agrupadas en doce conjuntos en relación al tema y a las unidades de diseño presentes. Las vasijas Famabalasto Negro Grabado exhiben una particular selección y adecuación de los temas y motivos presentes en la alfarería Santa María y Belén a través de un estilo concebido para destacarse con claridad de otros elementos del universo cerámico de la época, a la vez que se define como un nexo que pone en relieve las relaciones recíprocas entre ambos estilos mayoritarios de la época tardía, al incluir y enfatizar mediante las reiteraciones, ciertos temas y diseños comunes entre los mismos, y a su vez, entre estos y la metalurgia del bronce. El segmento incluye sendos catálogos ilustrados de las formas y diseños desplegados de las guardas.

Con el propósito de conocer las situaciones de uso y también la cronología del estilo, en los Capítulos 5 y $\mathbf{6}$ se analizan lo que llamamos los "contextos de la vida cotidiana" y los "contextos funerarios" respectivamente. En el primer caso se evalúa un total de 40 dataciones, de acuerdo con una metodología que jerarquiza la información a través del análisis de los niveles de asociación entre los fechados radiocarbónicos, los eventos datados y los conjuntos cerámicos. En el estudio de las asociaciones funerarias se aplicó también una distinción cualitativa según niveles de asociación. El análisis se aplica a 37 entierros mortuorios de infantes y adultos. A través del estudio crítico de los diversos niveles de asociación en estas tumbas, que en su mayoría fueron reutilizadas a lo largo de períodos de tiempo más o menos prolongados, se logra una segunda aproximación a su temporalidad con base en el conocimiento previo sobre la antigüedad y vigencia de uso de otros estilos cerámicos de época presentes en los sepulcros. Esta contextualización nos permite situar al estilo en una época histórica concreta que involucra los momentos intermedio-finales del Período Tardío, la época de expansión incaica y los primeros momentos del contacto hispano indígena en la zona. Como fenómeno regional se trata de un desarrollo que se expande hacia comienzos del siglo XIV con anterioridad a la influencia incaica en la zona y en conjunción con importantes fenómenos de transformaciones en las producciones cerámicas en la región. Con respecto a los ámbitos de empleo, se notó una presencia generalizada de fragmentos y vasijas en espacios domésticos, productivos y ceremoniales, por lo que planteamos que esta cerámica era una clase de objeto, quizás especial y estéticamente valorado, pero que podía ser observado y empleado en diversas situaciones destacadas o habituales de la vida cotidiana por un amplio segmento de la población.

En el Capítulo 7 se realiza una comparación estilística de placas metálicas y campanas de bronce tardías a través de la cual se destacan los elementos en común entre este grupo particular de bienes de la metalurgia del bronce y la cerámica Famabalasto Negro Grabado, y se señalan también aquellos rasgos por los que difieren. En especial las diferencias que se observaron tienen que ver con las frecuencias en el tratamiento del tema antropomorfo, en particular la cabeza cercenada, que se repite con insistencia en los metales y es en cambio muy rara en la cerámica. Postulamos la idea de que ambas tecnologías integraron un complejo estilístico, con relaciones recíprocas de carácter "icónico", formado por bienes de acceso social dispar, de carácter generalizado para las vasijas Famabalasto Negro Grabado, y limitado sólo a algunas personas o segmentos sociales para los valiosos bronces. De esta manera planteamos que este complejo actuó tanto en la integración colectiva, como también en la delineación de distinciones de índole jerárquicas, al participar toda la comunidad de su consumo, aunque de un modo diferencial, si consideramos que los metales 
especiales habrían circulado de manera más restringida, entre los miembros de elite de la comunidad.

Las conclusiones vertidas en cada capítulo se repasan en el Capítulo 8, donde se plantean discusiones, algunas conclusiones y varias líneas que futuros trabajos permitirán profundizar. Considerando las variantes tecnológicas, de morfología y de diseños, conjuntamente con la frecuencia de hallazgos en diferentes ámbitos, se postula provisoriamente la definición de variantes regionales en el estilo Famabalasto Negro Grabado sintetizada en la Figura 1. En la ilustración se representaron los cuatro sectores, definidos por la repetición de rasgos estilísticos, con líneas punteadas, y áreas superpuestas ya que esos ámbitos no significan la presencia exclusiva de dichos rasgos en cada sector. De hecho ciertos modelos de vasijas están extendidos por toda la región, sólo se destaca un énfasis en su expresión en esos espacios. El Área 1 comprende el valle Calchaquí, el Área 2, el centro y sur de Yocavil, el Área 3 incluye el norte de Yocavil, El Infiernillo, Tafí y el área pedemontana oriental de Tucumán. En al Área 4, la más austral, se incluyen los hallazgos de las zonas de Hualfín, Andalgalá y La Rioja.

Reflexionando sobre la dinámica del origen y desarrollo de nuevos estilos observamos cómo la estética que define a la alfarería Famabalasto Negro Grabado se relaciona con un resurgimiento de consignas visuales desde profundas raíces pre-tardías. Sin embargo, a la vez que evoca antiguas manifestaciones estilísticas, se configura como un estilo con marcados rasgos propios, por lo que lo calificamos como un estilo "vanguardista" que se nutre de viejos modelos, pero que no constituye copias de estos, resultando en una estética con definidos y novedosos rasgos propios. Planteamos que el Famabalasto Negro Grabado muestra semejanzas que lo vinculan con tradiciones presantamarianas, iniciando la tendencia de una época de cambios estilísticos en la que se reactivan elementos

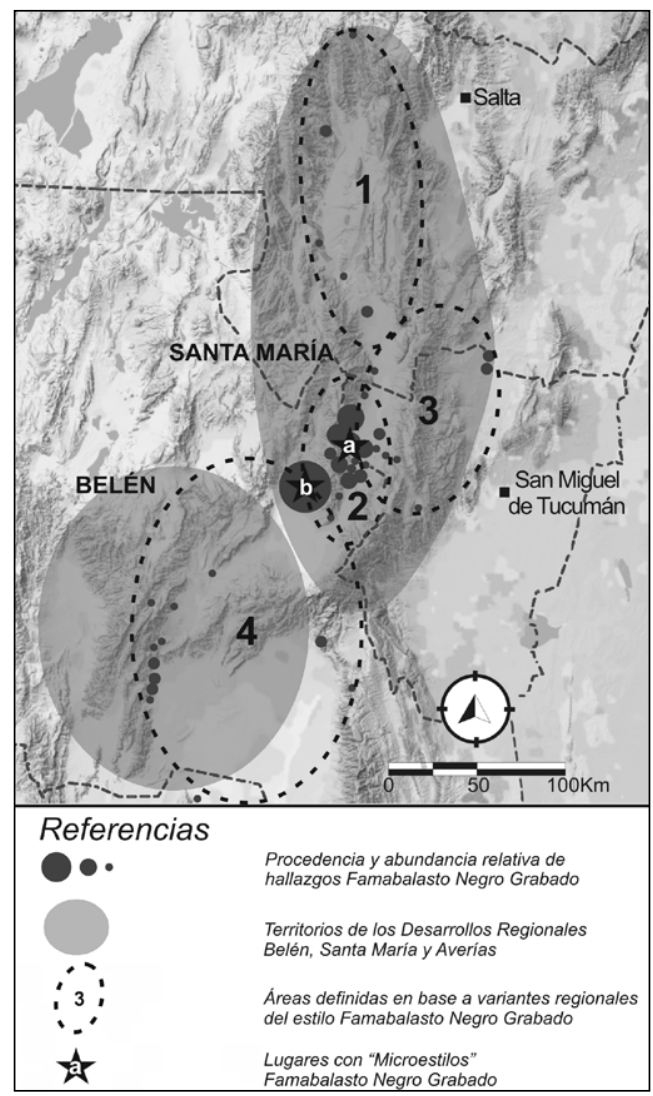

Figura 1. Propuesta de una distinción de VARIANTES regionales en el estilo Famabalasto Negro Grabado EN BASE A OBSERVACIONES SOBRE LAS FRECUENCIAS, LAS VARIANTES DE PASTAS, DE FORMAS Y DE DISEÑOS.

del simbolismo pretéritos del ámbito local y andino, como son las figuras del guerrerosacrificador, las cabezas cercenadas, las aves voladoras y los felinos, que vuelven a cobrar un notorio protagonismo luego de un período de desuso hacia finales del Período Tardío y en la época de expansión incaica. Una revivificación quizás vinculada con procesos locales o regionales de cambio en el escenario político.

Hacia comienzos del contacto hispano-indígena su elaboración y circulación comenzó a declinar. Mientras que la producción del Famabalasto Negro Grabado se desarticula$\mathrm{ba}$, nuevas producciones en la región retoman y modifican algunos de sus esquemas más repetidos, como puede apreciarse en algunos interesantes ejemplares de cerámicas Caspinchango, en los que similares pautas de 
manejo de los espacios de diseño e inclusive guardas muy populares en el, quizás para ese entonces, "antiguo" Famabalasto Negro Grabado se continuaron reproduciendo a través de una nueva estética en la que se seleccionan y acentúan los recursos de la abstracción.

Para finalizar este apretado resumen mencionaremos el contenido de los Apéndices que acompañan el texto:

I. Ilustraciones de piezas Famabalasto Negro Grabado (con calcos y fotografías de las vasijas que integran la muestra analizada)
II. Tabla de registro morfológico y métrico de piezas Famabalasto Negro Grabado

III. Catálogo de los contextos funerarios y objetos de los cementerios de Famabalasto (correspondientes a la IV Expedición Benjamín Muñiz Barreto, 1922, realizada por Vladimiro Weiser y Federico Wolters, con fotografías de los hallazgos e ilustraciones de los contextos funerarios

IV. Tabla de los hallazgos funerarios de la localidad de Famabalasto

V. Una recorrida por los cementerios de la localidad de Famabalasto

VI. El caso de las variantes Santa María Tricolor 
